\title{
Nowcasting: An R Package for Predicting Economic Variables Using Dynamic Factor Models
}

\author{
by Serge de Valk, Daiane de Mattos and Pedro Ferreira
}

\begin{abstract}
The nowcasting package provides the tools to make forecasts of monthly or quarterly economic variables using dynamic factor models. The objective is to help the user at each step of the forecasting process, starting with the construction of a database, all the way to the interpretation of the forecasts. The dynamic factor model adopted in this package is based on the articles from Giannone et al. (2008) and Banbura et al. (2011). Although there exist several other dynamic factor model packages available for $\mathrm{R}$, ours provides an environment to easily forecast economic variables and interpret results.
\end{abstract}

\section{Introduction}

Important economic decisions are made based on current and future conditions. Oftentimes, the variables used to measure such conditions are not available even for the recent past. This is, for instance, the case with US GDP that is published 45 days after the end of the quarter. Similarly, Brazilian GDP is published with a 60-day lag. There is therefore a need for forecasting the current value of given variables. To this end, Giannone et al. (2008) proposed a statistical model that allows quarterly variables, such as US GDP, to be forecast using a large set of monthly variables released with different lags. GDP forecasts for the current quarter are, furthermore, updated whenever new information is available. Different central banks have shown interest in this methodology, among them the European Central Bank (Angelini et al., 2008; Bańbura and Rünstler, 2011; Van Nieuwenhuyze et al., 2008), and the central banks of Ireland (D'Agostino et al., 2008), New Zealand (Matheson, 2010) and Norway (Aastveit and Trovik, 2012).

Factor models are designed to summarize the variation contained in a large dataset into only a few variables (Stock and Watson, 2006). In Giannone et al. (2008), the authors show how to reduce the information contained in dozens of monthly time series into only two dynamic factors. These two estimated factors, which are initially monthly, are then transformed into quarterly factors and used in a regression against GDP. Various other authors, such as Chauvet (2001); Marcellino et al. (2003); Forni et al. (2004); Boivin and Ng (2006); D'Agostino et al. (2006); Banbura et al. (2011); Dahlhaus et al. (2015); Stock and Watson (2016), have explored Dynamic Factor Models (DFMs) in time series forecasting and found promising results.

Given the publication lag of many variables, such as GDP, we can either forecast past, current or future values. In order to differentiate between those types of forecasts we adopt the terminology used in Giannone et al. (2008) and Banbura et al. (2011). Backcasting refers to forecasting the value of a yet unpublished variable for a past period, while nowcasting will be with respect to the current period. By way of illustration, suppose we want to forecast the GDP for the $2^{\text {nd }}$ quarter of 2018. If the exercise is made during the $2^{\text {nd }}$ quarter of 2018 , then the forecast is classified as nowcasting. However, if the current date is before the $2^{\text {nd }}$ quarter of 2018, then the term used is forecasting. Finally, if the date is after the $2^{\text {nd }}$ quarter of 2018 and the GDP has not yet been released, then the forecast is classified as backcasting.

The aim of the package nowcasting is to offer the tools for the R user to implement dynamic factor models. The different steps in the forecasting process and the associated functions within the package are based on the literature. We have chosen to divide the process into 4 main steps: 1) constructing a dataset; 2) defining the model's initiation parameters; 3) forecasting; 4) presenting results. This particular division will be maintained in most sections.

This brings us to the article's sections that are organized as follows: 1) the theoretical framework is introduced; 2) the functions of our package are presented; 3) working examples of how to nowcast Brazilian GDP and of the New York FED nowcasting are given; 4 ) and finally the last section concludes with some considerations. 


\section{Methodology}

\section{Dynamic Factor Model}

Let $x_{t}=\left(x_{1, t}, x_{2, t}, \ldots, x_{N, t}\right)^{\prime}$ be the vector representing $N$ monthly time series transformed to satisfy the weak stationarity assumption. The general specification of the dynamic factor model is given by:

$$
\begin{aligned}
& x_{t}=\mu+\Lambda f_{t}+\varepsilon_{t} \\
& f_{t}=\sum_{i=1}^{p} A_{i} f_{t-i}+B u_{t}, \quad u_{t} \sim \text { i.i.d.N }\left(0, I_{q}\right)
\end{aligned}
$$

In equation (1), the variables $x_{t}$ are expressed as a function of an intercept $\mu$ and $r$ unobserved common factors $f_{t}$. Since all variables $x$ will later be demeaned, one may drop the unconditional means $\mu$. The variables $x_{t}$ will be loaded into the unobserved factors $f_{t}$ through $\Lambda$. Equation (2) imposes the structure of a $\operatorname{VAR}(\mathrm{p})$ process on the factors $f_{t}$. Both $\varepsilon_{t}$ and $u_{t}$ are normal, allowing the use of the Kalman Filter. Furthermore, the vector of idiosyncratic component $\varepsilon_{t}$ is unrelated to $u_{t}$ at all lags, i.e., $E\left[\epsilon_{t} u_{t-k}^{\prime}\right]=0$ for any $\mathrm{k}$. An interesting feature of equation (2) is that the number of shocks $q$ to the factors need not be equal to the number of factors $r$. Structural breaks or lead/lag relationships of the $r$ factors with $q$ common shocks may motivate such a modeling choice (see Stock and Watson (2016) for more information).

In the so-called exact dynamic factor model, the error components from equation (1) are assumed to be mutually uncorrelated at all lags, i.e., $E\left[\varepsilon_{i, t} \varepsilon_{j, s}\right]=0$ for $i \neq j$. However, following Banbura et al. (2011), the error term could be modeled as an AR( $\left.\mathrm{p}^{\prime}\right)$ process:

$$
\varepsilon_{i, t}=\sum_{j=1}^{p^{\prime}} \alpha_{i, j} \varepsilon_{i, t-j}+e_{i, t}, \quad e_{i, t} \sim \text { i.i.d.N }\left(0, \sigma_{i}^{2}\right)
$$

where $E\left[e_{i, t} e_{j, s}\right]=0$ for $i \neq j$.

Following is an example, in matrix form, of equation (2) of the model for orders $r=2, p=2$ and $q=2$.

$$
\begin{gathered}
{\left[\begin{array}{c}
f_{1, t} \\
f_{2, t} \\
f_{1, t-1} \\
f_{2, t-1}
\end{array}\right]=\left[\begin{array}{cccc}
a_{1,1}^{1} & a_{1,2}^{1} & a_{1,1}^{2} & a_{1,2}^{2} \\
a_{2,1}^{1} & a_{2,2}^{1} & a_{2,1}^{2} & a_{2,2}^{2} \\
1 & 0 & 0 & 0 \\
0 & 1 & 0 & 0
\end{array}\right]\left[\begin{array}{l}
f_{1, t-1} \\
f_{2, t-1} \\
f_{1, t-2} \\
f_{2, t-2}
\end{array}\right]+\left[\begin{array}{cc}
b_{1,1} & b_{1,2} \\
b_{2,1} & b_{2,2} \\
0 & 0 \\
0 & 0
\end{array}\right]\left[\begin{array}{l}
u_{1, t} \\
u_{2, t}
\end{array}\right]} \\
F_{t}=\left[\begin{array}{cc}
A_{1} & A_{2} \\
I_{2} & 0
\end{array}\right] F_{t-1}+B u_{t}
\end{gathered}
$$

\section{Quarterly and monthly variables}

In order to predict a quarterly variable using monthly data, we construct a partially observed monthly counterpart of the quarterly variable as proposed in Mariano and Murasawa (2003). This allows, for instance, quarterly GDP to be explained by monthly variables. Continuing with this example, let $Y_{t}^{M}$ be the level of the unobservable monthly GDP level and $Y_{t}^{Q}$ the quarterly value of GDP for the partially observable monthly series. As is usual in the literature, we let quarterly GDP be observable in the third month of the quarter.

$$
Y_{t}^{Q}=\left\{\begin{array}{lc}
Y_{t}^{M}+Y_{t-1}^{M}+Y_{t-2}^{M}, & t=3,6,9, \ldots \\
\text { unobserved } & \text { otherwise }
\end{array}\right.
$$

The above accounting rule states that the quarterly GDP flow is equal to the sum of the monthly flows. Looking at the quarterly change, $y_{t}^{Q}=Y_{t}^{Q}-Y_{t-3}^{Q}{ }^{1}$, it is easy to show that it can be expressed

\footnotetext{
${ }^{1}$ The aggregation scheme, and ensuing weights used for aggregating the monthly series, may differ according to the order of the difference taken. In the paper of Mariano and Murasawa (2003), the example is of a first difference of quarterly $\log$ GDP, which corresponds to a quarterly growth rate. In the case of an annual growth rate, $\Delta_{12} \log \left(Y_{t}^{Q}\right)=\log \left(Y_{t}^{Q}\right)-\log \left(Y_{t-12}^{Q}\right)$, the aggregation weights would be different. Such cases are not considered here.
} 
as a function of the differences of the monthly variable, $y_{t}=Y_{t}^{M}-Y_{t-1}^{M}$, by using equation (6):

$$
\begin{aligned}
y_{t}^{Q} & =Y_{t}^{Q}-Y_{t-3}^{Q} \\
& =Y_{t}^{Q}+Y_{t-1}^{Q}-Y_{t-1}^{Q}+Y_{t-2}^{Q}-Y_{t-2}^{Q}-Y_{t-3}^{Q} \\
& =y_{t}+2 y_{t-1}+3 y_{t-2}+2 y_{t-3}+y_{t-4}, \quad t=6,9, \ldots
\end{aligned}
$$

Suppose that the variable of interest is a quarterly rate of change, $x_{t}^{Q}$, defined as:

$$
x_{t}^{Q} \equiv \log \left(Y_{t}^{Q}\right)-\log \left(Y_{t-3}^{Q}\right)
$$

Stating the approximation between the arithmetic and geometric means we have:

$$
\frac{1}{3}\left[Y_{t}^{M}+Y_{t-1}^{M}+Y_{t-2}^{M}\right] \approx \sqrt[3]{Y_{t}^{M} Y_{t-1}^{M} Y_{t-2}^{M}}
$$

Combining equations (8) and (9) we obtain the approximation from Mariano and Murasawa (2003) that expresses the quarterly growth rate of GDP as a function of the unobservable monthly growth rates $x_{t}^{M}$ :

$$
x_{t}^{Q} \approx \frac{1}{3}\left[x_{t}^{M}+2 x_{t-1}^{M}+3 x_{t-2}^{M}+2 x_{t-3}^{M}+x_{t-4}^{M}\right]
$$

Suppose that the unobserved monthly growth rate $x_{t}^{M}$ also admits the same factor representation as in equation (1) with loadings $\Lambda_{Q}$, then the quarterly GDP growth rate, $x_{t}^{Q}$, can be expressed as a function of monthly factors.

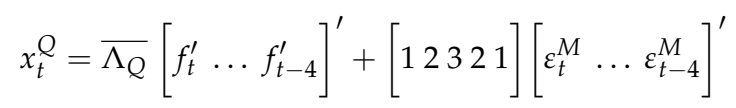

where $\overline{\Lambda_{Q}}=\left[\Lambda_{Q} 2 \Lambda_{Q} 3 \Lambda_{Q} 2 \Lambda_{Q} \Lambda_{Q}\right]$ is a restricted matrix of loadings on the factors and their lags. Note that the errors are normal in the exact dynamic factor model or have an AR(1) structure as in Banbura et al. (2011).

\section{Determining the number of factors and shocks to the factors}

We follow the papers by Bai and Ng (2002) and Bai and Ng (2007) to respectively define 1) the number $r$ of factors in equation (1) and 2) the number of shocks $q$ to the factors in equation (2).

Let $V\left(r, \hat{F}^{r}\right)$ be the sum of squared residuals when $r$ factors are estimated using principal components. The the information criteria can then be written as follows:

$$
\begin{gathered}
I C_{r 1}(r)=\ln \left(V\left(r, \widehat{F}^{r}\right)\right)+r\left(\frac{N+T}{N T}\right) \ln \left(\frac{N T}{N+T}\right) \\
I C_{r 2}(r)=\ln \left(V\left(r, \widehat{F}^{r}\right)\right)+r\left(\frac{N+T}{N T}\right) \ln (\min \{N, T\}) \\
I C_{r 3}(r)=\ln \left(V\left(r, \widehat{F}^{r}\right)\right)+r\left(\frac{\ln (\min \{N, T\})}{\min \{N, T\}}\right)
\end{gathered}
$$

The chosen number of factors $r^{*}$ will then correspond to $\arg \min _{r} I C_{r i}(r)$, for $i \in\{1,2,3\}$. Equations (12), (13), and (14) are asymptotically equivalent, but may nevertheless give significantly different results for finite samples. To this effect, observe that the penalty in equation (13) is highest when considering finite samples.

The number of shocks q can be lower than the number of factors $r$. Once the number of factors is determined, we use an information criterion from Bai and $\mathrm{Ng}$ (2007) to estimate the number of shocks $q$ in equation (2). Let $\widehat{F}_{t}$ be the $r$ factors estimated using principal components and let $\widehat{u}_{t}$ be the residuals from the VAR $A(l) \widehat{F}_{t}=\widehat{u}_{t}$. The idea is to check whether the eigenvalues of the variance-covariance matrix $\widehat{\Sigma}_{u}$ are different from 0 . Numerically, we will therefore want to test whether a given eigenvalue is below a predefined tolerance level.To this end, define the eigenvalues $c_{1}>c_{2} \geq \ldots \geq c_{r} \geq 0$ of $\widehat{\sum}_{u}$ 
and define the $\mathrm{k}^{\text {th }}$ normalization of the $\mathrm{k}+1^{\text {th }}$ eigenvalue

$$
\widehat{D}_{k}=\left(\frac{c_{(k+1)}^{2}}{\sum_{j=1}^{r} c_{j}^{2}}\right)^{1 / 2}
$$

Then for some $0<m<\infty$ and $0<\delta<1 / 2$ that set the tolerance level, define the vector $\mathrm{K}$

$$
K=\left\{k: \widehat{D}_{k}<m / \min \left[N^{1 / 2-\delta}, T^{1 / 2-\delta}\right]\right\}
$$

where the estimated number of shocks to the factors will be $\hat{q}=\min \{k \in K\}$. This estimator will converge in probability towards the real number of shocks given that $r$ is the real number of factors.

\section{Estimation}

We will describe two methodologies for estimating dynamic factors: Two-Stage and ExpectationMaximization.

1. Two-Stage: This approach is described in Giannone et al. (2008) and refers to the exact DFM. In the first stage, the parameters of the matrices $\Lambda$ and $f_{t}$ are estimated by Principal Components Analysis (PCA) using a standardized, balanced panel $\left(\bar{X}_{t}\right)$, in which there are no missing values and outliers. Standardization is important as PCA is not scale invariant. The estimators $\widehat{\Lambda}$ and $\widehat{f}_{t}$ can be obtained by solving the following optimization problem:

$$
\min _{f_{1}, \ldots, f_{T}, \Lambda} \frac{1}{N T} \sum_{t=1}^{T}\left(\bar{X}_{t}-\Lambda f_{t}\right)^{\prime}\left(\bar{X}_{t}-\Lambda f_{t}\right) \quad \text { s.t. } \quad N^{-1} \Lambda^{\prime} \Lambda=I_{r}
$$

The estimator for the variance and covariance matrix for $\varepsilon_{t}$ is then given by

$$
\widehat{\Psi}=\operatorname{diag}\left(\frac{1}{T} \sum_{t=1}^{T}\left(\bar{X}_{t}-\widehat{\Lambda} \widehat{f}_{t}\right)\left(\bar{X}_{t}-\widehat{\Lambda} \widehat{f}_{t}\right)^{\prime}\right)
$$

According to Stock and Watson (2011), the solution to (17) is to set $\widehat{\Lambda}$ equal to the eigenvectors of the variance and covariance matrix of $\bar{X}_{t}$ associated with the $r$ largest eigenvalues, from which it follows that the vector $\widehat{f}_{t}$ is the $r$ first principal components of $\bar{X}_{t}$. The coefficients of the matrix $A_{i}, i=1,2, \ldots, p$, from equation (2), are estimated by OLS regression of $f_{t}$ on $f_{t-1}, \ldots, f_{t-p}$. Finally, $B B^{\prime}$ is estimated as the covariance matrix of the residuals of this regression.

In the second stage, Kalman smoothing (Durbin and Koopman, 2012) is used to re-estimate the factors for the unbalanced panel $x_{t}$ considering the parameters obtained in the previous step. There are some R packages that implemented the Kalman smoothing (Tusell, 2011). However, for convenience, in the nowcasting package, we used the routine provided by Giannone et al. (2008). Furthermore, two options are provided when estimating the factors:

- No aggregation: No bridge equation, to obtain (19), is needed if both the dependent and the explanatory variables are monthly indicators. Hence, the aggregation procedure as set out in Mariano and Murasawa (2003) is not required. Similarly, if the explanatory variables have been transformed to represent quarterly quantities, the same aggregation procedure does not need to be implemented again on the factors.

- With aggregation: This option is relevant when having a dependent variable y of lower frequency than the explanatory variables. Factors are estimated using the monthly explanatory variables $x$, after which the transformation from Mariano and Murasawa (2003) is applied in order to obtain factors representing quarterly quantities. Those will be used to forecast the dependent variable in the bridge equation (19).

$$
y_{t}=\beta_{0}+\beta^{\prime} \widehat{f}_{t}+e_{t}
$$

The parameters of equation (19) are estimated by OLS, and the forecast for $y_{t+h}$ is given by

$$
\widehat{y}_{t+h}=\widehat{\beta_{0}}+\widehat{\beta}^{\prime} \widehat{f}_{t+h}
$$

2. Expectation-Maximization: This estimation method is able to deal with arbitrary patterns of missing values as shown in Bańbura and Modugno (2014). It is therefore less restrictive than the Two-Stage method with regards to the frequencies of the variables and allows for a mixed frequency database. Following Banbura et al. (2011), factors can be defined for different 
subgroups of variables and no longer all need to be global as in the Two-Stage estimation method. Below, we illustrate a case where three factors are partitioned into three groups (global, real and nominal) as in Banbura et al. (2011). Rewriting equation (1) accordingly gives equation (21). As opposed to the Two-Stage estimation method that builds on an exact dynamic factor model, the error term is defined as an AR(1) process. A more restrictive assumption than the Two-Stage method is that the number of shocks to the factors $q$ is set equal to the number of factors $r$.

$$
x_{t}=\mu+\left(\begin{array}{ccc}
\Lambda_{N, G} & \Lambda_{N, N} & 0 \\
\Lambda_{R, G} & 0 & \Lambda_{R, R}
\end{array}\right)\left(\begin{array}{c}
f_{t}^{G} \\
z f_{t}^{N} \\
f_{t}^{R}
\end{array}\right)+\varepsilon_{t}
$$

where

$$
\begin{gathered}
\left(\begin{array}{ccc}
\Lambda_{N, G} & \Lambda_{N, N} & 0 \\
\Lambda_{R, G} & 0 & \Lambda_{R, R}
\end{array}\right)=\Lambda \\
\left(\begin{array}{c}
f_{t}^{G} \\
f_{t}^{N} \\
f_{t}^{R}
\end{array}\right)=f_{t}
\end{gathered}
$$

The global factor is estimated considering all the explanatory variables, while the estimates of the nominal and real factors only consider variable classified, respectively, as nominal and real. The parameter $\mu$ is a vector of constants of dimension N. As previously mentioned, the alternative proposed by Banbura et al. (2011) to the exact DFM, allows for serial autocorrelation among the error of equation (1) along an $A R(1)$ process:

where $E\left[e_{i, t} e_{j, s}\right]=0$ for $i \neq j$.

$$
\varepsilon_{i, t}=\alpha_{i} \epsilon_{i, t-1}+e_{i, t}, \quad e_{i, t} \sim \text { i.i.d.N }\left(0, \sigma_{i}^{2}\right)
$$

In this model, the parameters, the unobserved common factors and the missing values are estimated through the Expectation-Maximization algorithm, which uses the following recursive structure:

- E-step: The conditional expectation of the likelihood function is calculated using the estimates of the static parameters $(\theta)$ from the previous iteration, $\theta_{j}$;

- M-step: The new parameters, $\theta_{j+1}$ are estimated by maximizing the likelihood function from the previous step with respect to $\theta$.

Convergence is achieved when the absolute change in the value of the log-likelihood function is less than $10^{-4}$, the tolerance level used for this algorithm. The recursive process starts with the PCA estimates given in Giannone et al. (2008) (first stage of the Two-Stage method).

\section{The $\mathrm{R}$ package}

\section{Working on the dataset}

The first step in the nowcasting process is to prepare the data in a way that is compatible with the proposed models and estimation methods. One of the motivations of the presented models is the forecasting improvements that can be achieved by using higher frequency variables. More specifically, the gains that can be obtained in using monthly variables to forecast quarterly series. Hence, all functions require monthly mts objects. In practice, the quarterly variables are usually represented as monthly variables for which the last month of the quarter is observed. As illustrated in the working examples, such straightforward transformations from one frequency representation to another can be achieved by using the functions qtr2month() or month2qtr().

With regards to the estimation methods, different inputs may have to be provided. As a matter of fact, the Two-Stage method is more restrictive on the format of the variables as it depends on principal components in the first stage. This requires a strategy to deal with missing values, which are not part of the jagged edge, beforehand. Giannone et al. (2008) propose to replace such missing values with the median of the series that are then smoothed with a moving average. Since such a strategy assigns a value that is independent of the information contained in other contemporaneous variables, it is advisable to exclude series with many missing values. The EM algorithm, however, is able to deal with missing values in a way that uses the information contained in other variables and might therefore not require discarding such variables. Finally, independently of the estimation method, stationary series are required. The usual transformations for making time series stationary and the different strategies 
to deal with missing values have been included in the function Bpanel() that prepares the database for the nowcasting function. Since these choices require careful attention, the function Bpanel( $)$ is explained in further detail.

Bpanel (base, trans, NA.replace $=$ TRUE, aggregate $=$ FALSE, $k \cdot m a=3$, na $\cdot$ prop $=1 / 3, h=12$ )

trans is a vector indicating the transformations to be applied to the variables. For most cases, the available transformations are sufficient to make economic variables stationary. The transformation must be specified by using one of the following values for the argument trans:

trans $=0$ : the observed series is preserved;

trans $=1$ : monthly rate of change: $\frac{x_{i, t}-x_{i, t-1}}{x_{i, t-1}}$;

trans $=2$ : monthly difference: $x_{i, t}-x_{i, t-1}$;

trans $=3$ : monthly difference in year-over-year rate of change:

$$
\frac{x_{i, t}-x_{i, t-12}}{x_{i, t-12}}-\frac{x_{i, t-1}-x_{i, t-13}}{x_{i, t-13}}
$$

trans $=4:$ monthly difference in year-over-year difference:

$$
\left(x_{i, t}-x_{i, t-12}\right)-\left(x_{i, t-1}-x_{i, t-13}\right) .
$$

trans $=5:$ year difference:

$$
\left(x_{i, t}-x_{i, t-12}\right)
$$

trans $=6$ : year-over-year rate of change:

$$
\frac{x_{i, t}-x_{i, t-12}}{x_{i, t-12}}
$$

trans $=7$ : quarterly rate of change

$$
\frac{x_{i, t}-x_{i, t-3}}{x_{i, t-3}}
$$

NA. replace is a boolean to determine whether missing values should be replaced (NA. replace = TRUE) or not (NA. replace $=$ FALSE).

aggregate is a boolean to indicate whether to aggregate the monthly variables to represent quarterly quantities. If TRUE the aggregation is made following the approximation of Mariano and Murasawa (2003).

k. ma is a numeric representing the degree of the moving average correction if NA. replace = TRUE.

na.prop is a number between 0 and 1 indicating the ratio of missing observations to the total number of observations beyond which series will be discarded. The default is $1 / 3$, meaning that if more than $1 / 3$ of the observations are missing the series will be discarded from the database.

$\mathrm{h}$ indicates how many periods should be added to the database. Default is 12 . Those missing values will be predicted with the function nowcast().

\section{Determining the number of factors and shocks to the factors}

As explained in the section on parameter estimation, the package offers different functions to estimate the number of factors $r$ and of idiosyncratic shocks $q$ of equations (1) and (2) respectively.

1. Function ICfactors() estimates the number of factors $r^{*}$ according to an information criterion. The argument $x$ is a balanced panel and $r$ max is an integer representing the maximum number of factors for which the information criterion should be calculated. The default value is 20. type indicates which of the information criterion from Bai and $\operatorname{Ng}(2002)$ to use. type $\in\{1,2,3\}$ with the default being 2 as explained in the methodological section. If $x$ is not a balanced panel, the function will delete rows with missing values in order to use principal components.

$\operatorname{ICfactors}(x, \quad r m a x=20$, type $=2)$

2. Function ICshocks() estimates the number of idiosyncratic shocks given a number $r$ of factors according to the information criterion introduced in the previous section. The argument $x$ is a balanced panel. delta and $m$ are parameters of the information criterion, where $0<m<\infty$ and 
$0<\delta<1 / 2$. The default values are those from Bai and $\mathrm{Ng}$ (2007): $m=1$ and $\delta=0.1$. If the number of factors $r$ is not specified it will be defined according to ICfactors $(x, r$ max $=20$, type $=2$ ). $p$ is the number of lags in the VAR of equation (2). If not specified, the default is the lowest most occurring value from the information criteria used within the function VARselect() from the package vars.

ICshocks (x, $r=$ NULL, $p=$ NULL, delta $=0.1, m=1)$

\section{Forecasts}

An important feature of factor models is the dimensionality reduction of (many) original variables into a few common factors. Hence, the target variable y will be expressed as a function of a few factors extracted from the explanatory variables. This motivated the choice of the inputs for the nowcast() function. The formula format, which is well known to R users, captures this idea as formula $=\mathrm{y} \sim$. can be understood as the projection of $y$ on the information contained in the dataset. The model's parameters are estimated according to the selected method (2s, 2s_agg and EM, which correspond, respectively, to "two-stage", "two-stage with factor aggregation" and "Expectation-Maximization algorithm") described in the section on estimation. The number $r$ of dynamic factors, the number $q$ of shocks to the factors, and the lag order $p$ of the factors are determined beforehand as shown in the previous subsection. The argument blocks can be used with the EM method to estimate factors for different subgroups of variables. Finally, the argument frequency is necessary for all methods in order to identify the frequency of the variables.

nowcast (formula, data, $q=$ NULL, $r=$ NULL, $p=$ NULL, method $=$ 'EM', blocks $=$ NULL, frequency $=$ NULL)

In the first two methods (2s and 2s_agg), the factors are calculated based on the monthly variables, on which the dependent variable y will be regressed. The difference between $2 \mathrm{~s}$ and $2 \mathrm{~s}$ _agg is that for the latter the monthly factors are transformed into quarterly quantities while in the former no such aggregation is used. A linear regression (bridge equation if $\mathrm{y}$ is quarterly) of $\mathrm{y}$ on the factors allows the former to be forecast.

In the third method (EM) no bridge equation is needed, as opposed to the Two-Stage method. In practice, the algorithm will estimate all the missing values respecting the restrictions imposed by equation (11). The forecasts of quarterly time series are defined as the estimated values of the third month of the out of sample quarters. As opposed to the Two-Stage method, the number of common shocks q can not be specified and is assumed to be equal to $r$, the number of factors in each block.

\section{Analyzing the results}

The function nowcast. plot() allows to plot several outputs from the function nowcast().

nowcast.plot (out, type $=$ "fcst")

The argument out is the output from the function nowcast(). The argument type can be chosen from the list \{"fcst","factors","eigenvalues","eigenvectors"\}:

- "fcst": shows the y variable and its forecasts in sample and out of sample.

- "factors": shows all the estimated factors.

- "eigenvalues": indicates how much of the variability in the dataset is explained by each factor.

- "eigenvectors": shows the importance of each variable in the first factor.

\section{A working example of the Two-Stage method: nowcasting Brazilian GDP}

\section{Constructing the dataset}

In this example we showcase how to nowcast Brazilian GDP using the Two-Stage estimation method. Most of the variables of interest can be downloaded from the Brazilian central bank using the function BETSget() from the package BETS. The variables and the associated codes can be found on the Brazilian central bank's website ${ }^{2}$. For the sake of simplicity we have included the database, and all relevant information within the package ${ }^{3}$.

\footnotetext{
${ }^{2}$ see http://www4.bcb.gov.br/pec/series/port/aviso.asp

${ }^{3}$ The database is a random sample of 100 variables from our own database
} 
$>$ library(nowcasting)

$>\operatorname{data}(B R G D P)$

For this example we will construct a pseudo real-time dataset, using the function $\operatorname{PRTDB}()$. Some variables, such as GDP, suffer revisions over time. Since we do not take revisions into account, we refer to such datasets as pseudo real-time (as opposed to vintages). The (approximate) delays in days are included in the BRGDP object and will be used to define if observations were available at a specific moment in time. The dataset is then treated for outliers and missing values that are not part of the jagged edges of the data, i.e., that are not due to the different publication lags of the variables. This is achieved through the function Bpanel(). Unless otherwise specified by the user, the function will also discard series with over $1 / 3$ missing values.

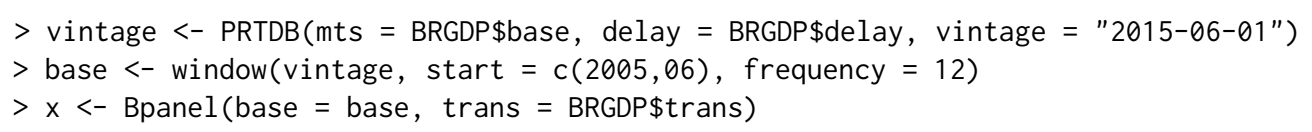

The function month2qtr() transforms monthly time series into quarterly ones. In this case we want to use the value of the third month as the quarterly value.

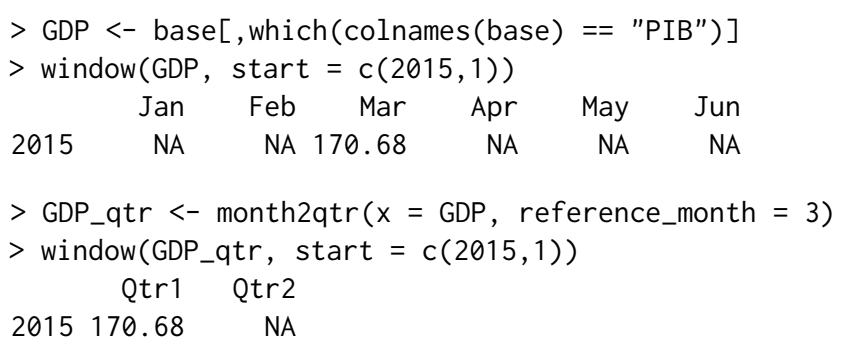

The quarterly GDP indicator, in this example, is an index representing the seasonal quarterly product. $\Delta_{4} Y_{t}$ deals with seasonality, while $\Delta \Delta_{4} Y_{t}$ is necessary to obtain a stationary time series. To test the latter, one could look at tests for unit roots or serial auto correlation that are included in many $\mathrm{R}$ packages.

$>y<-\operatorname{diff}(\operatorname{diff}($ GDP_qtr,4))

$>\mathrm{y}<-\operatorname{qtr} 2 \operatorname{month}(\mathrm{y})$

\section{Determining the number of factors and shocks}

The dataset $x$, which now only posses jagged edges, is well suited for the information criteria that make use of principal components. The estimated number of factors is given by the function ICfactors(). As explained in the previous section, the information criteria might give different results for finite samples.

$>$ ICR1 <- ICfactors $(x=x$, type $=1)$

\section{ICR1}

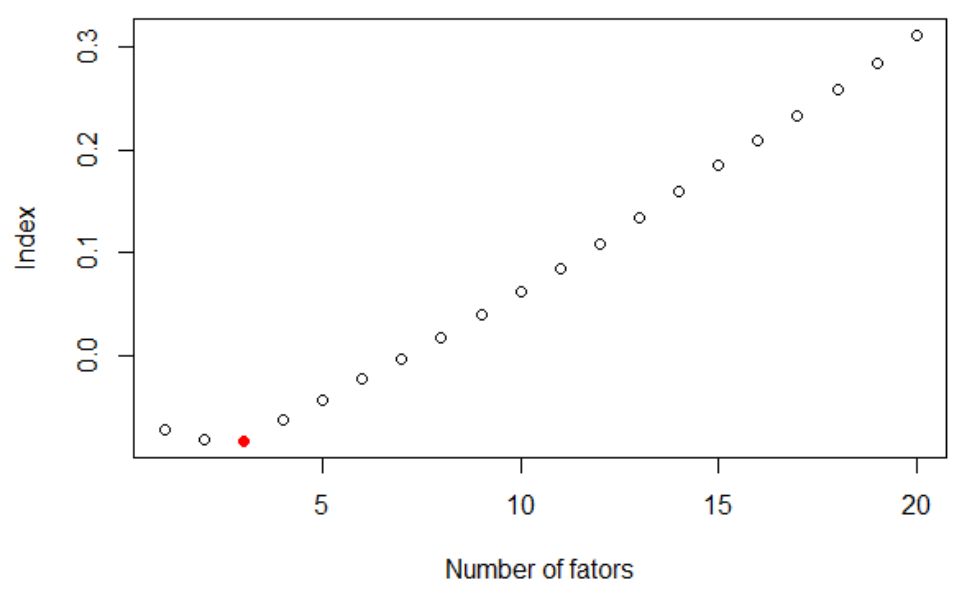


$>$ ICR2 $<-$ ICfactors $(x=x$, type $=2)$

ICR2

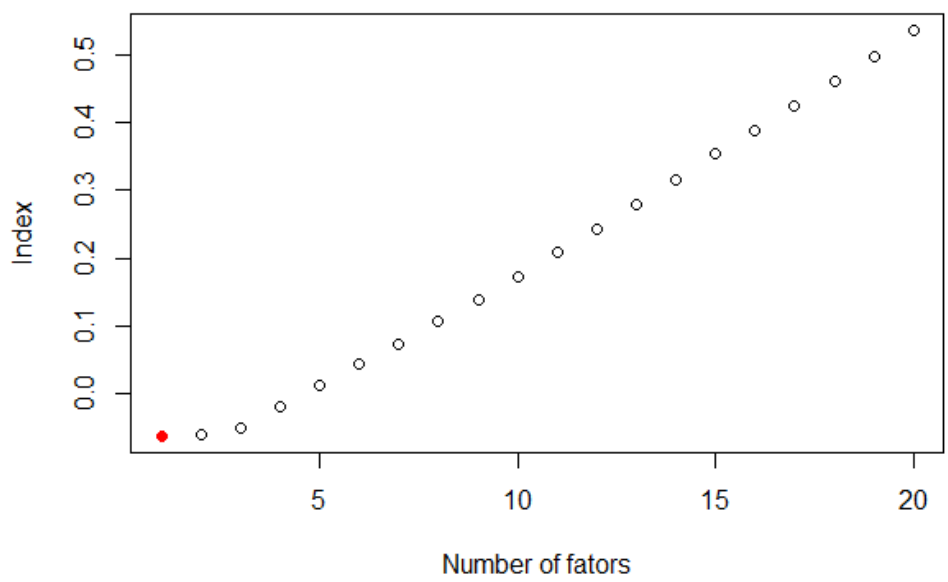

Finally, given the chosen number of factors for our model, we can use an information criterion for determining the number of shocks to the factors.

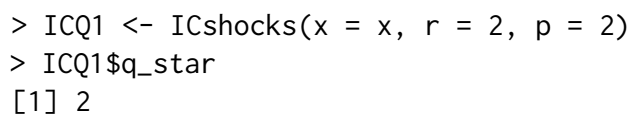

\section{Forecasts}

Let the object data be a monthly mts object where the first column is a partially observable stationary GDP series ( $y$ ) and the remaining columns a balanced panel of stationary time series (x). The frequency vector will be determined by the quarterly GDP series and the remaining monthly series. In this example the factors will be aggregated to obtain quarterly quantities by setting method = "2s_agg".

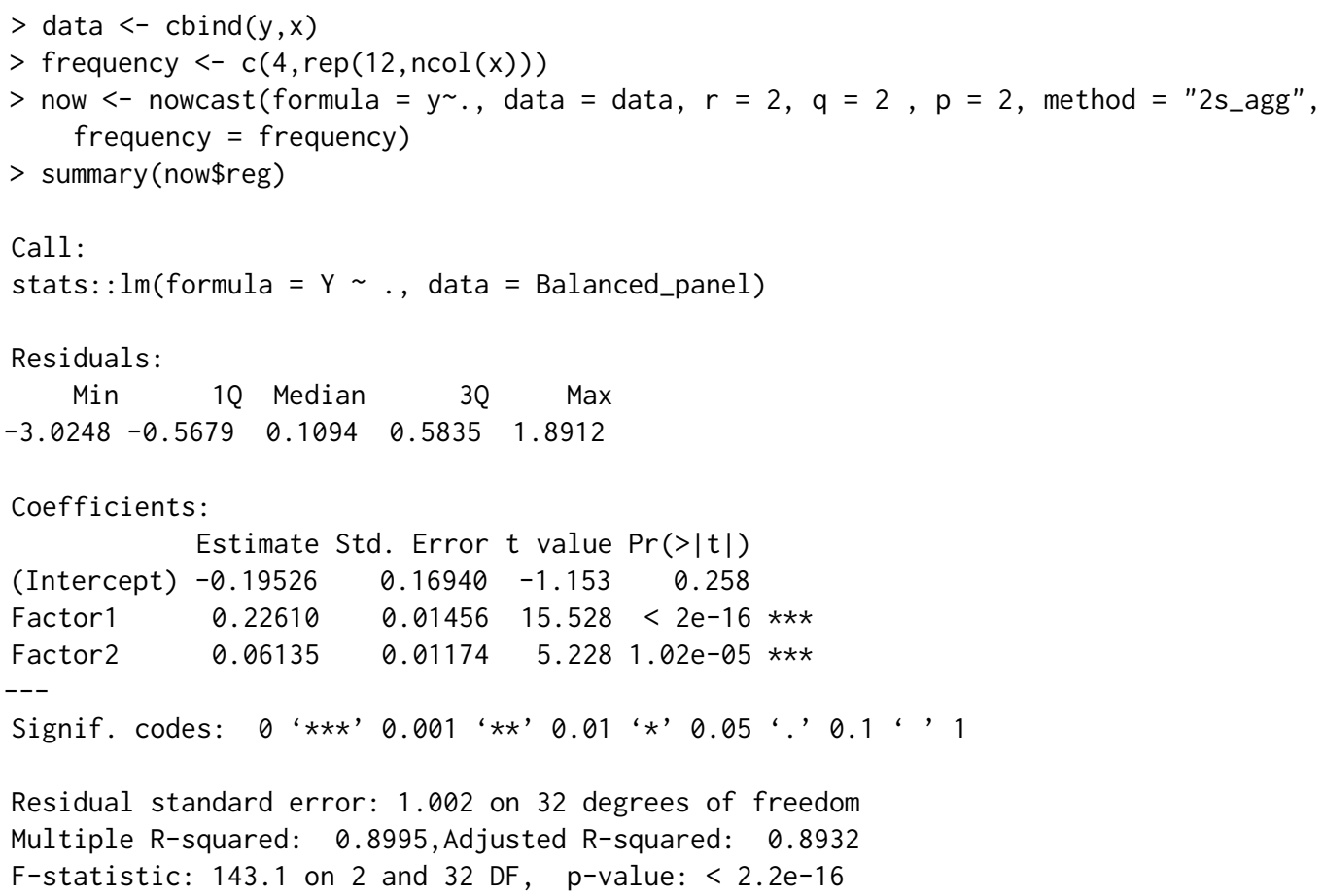




\section{Results}

The function nowcast.plot () enables the user to visualize some of the results. Say, for instance, that we want to look at fitted values and out-of-sample forecasts. This can be achieved by setting the type to "fcst". We might also want to look at the eigenvalues of the normalized variance-covariance matrix of our balanced panel or at how variables enter the first factor.

$>$ nowcast.plot (now, type $=" f c s t ")$

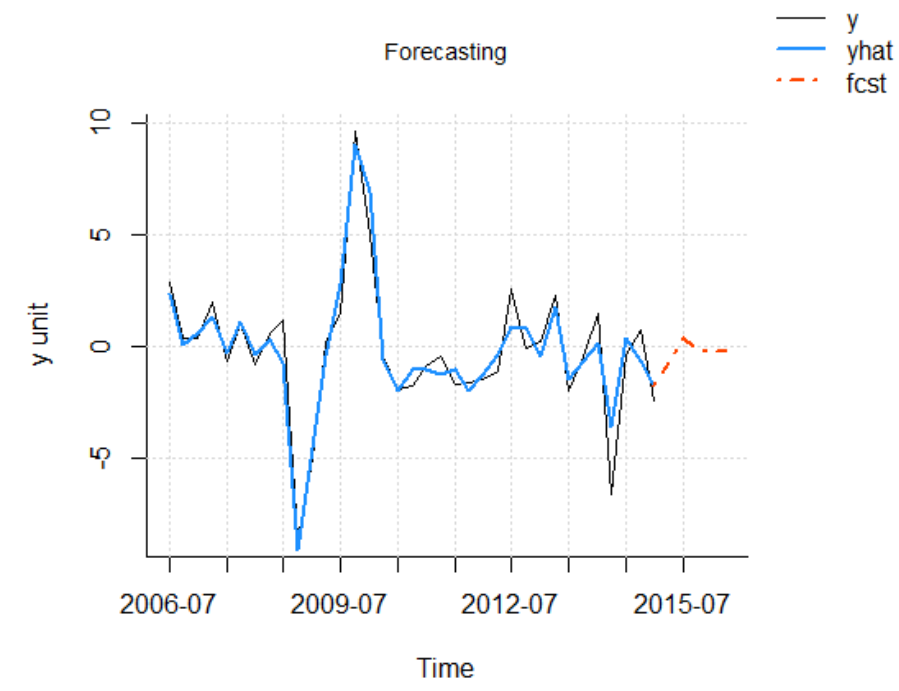

$>$ nowcast.plot (now, type $=$ "eigenvalues")

eigenvalues: percentage variance

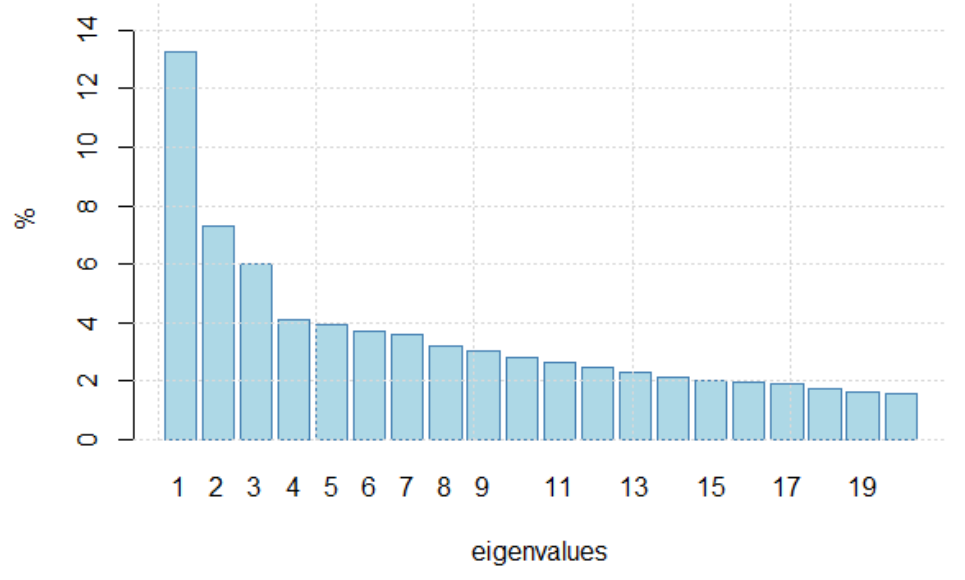

$>$ nowcast.plot (now, type $=$ "eigenvectors") 


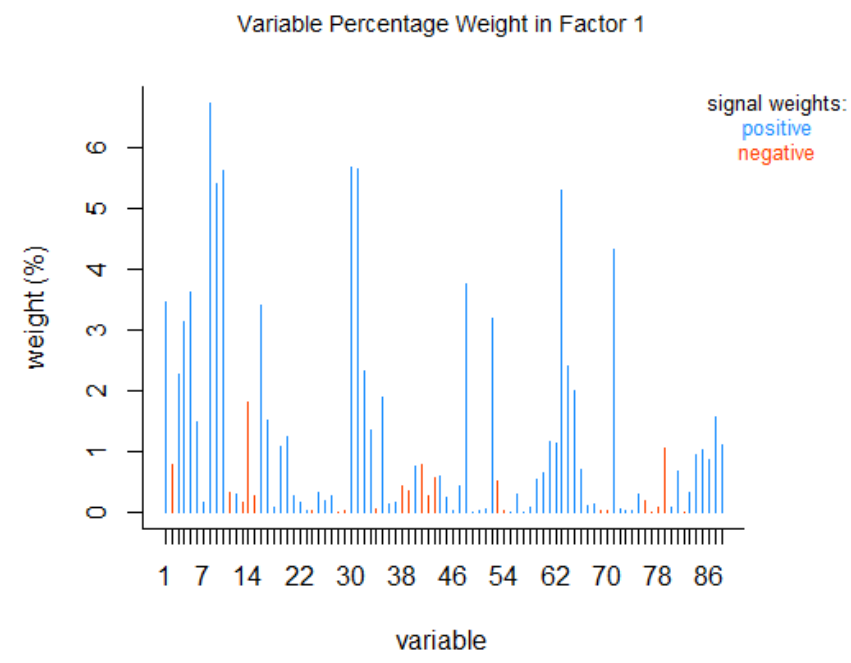

Up until now, we have been forecasting GDP after transforming it into a stationary variable. We might want to transform the former back into a level variable in order to forecast the actual growth rate. Remember that we transformed GDP according to

$$
\begin{aligned}
\operatorname{diff}\left(\operatorname{diff}\left(G D P_{t}, 4\right)\right) & =\left(G D P_{t}-G D P_{t-4}\right)-\left(G D P_{t-1}-G D P_{t-5}\right) \\
& =G D P_{t}+G D P_{t-5}-G D P_{t-1}-G D P_{t-4}
\end{aligned}
$$

that can be rewritten as

$$
G D P_{t}=\operatorname{diff}\left(\operatorname{diff}\left(G D P_{t}, 4\right)\right)-G D P_{t-5}+G D P_{t-1}+G D P_{t-4}
$$

Equation (26) gives us the forecast of the new quarter GDP level. The variable BRGDP\$GDP is the non-stationary GDP.

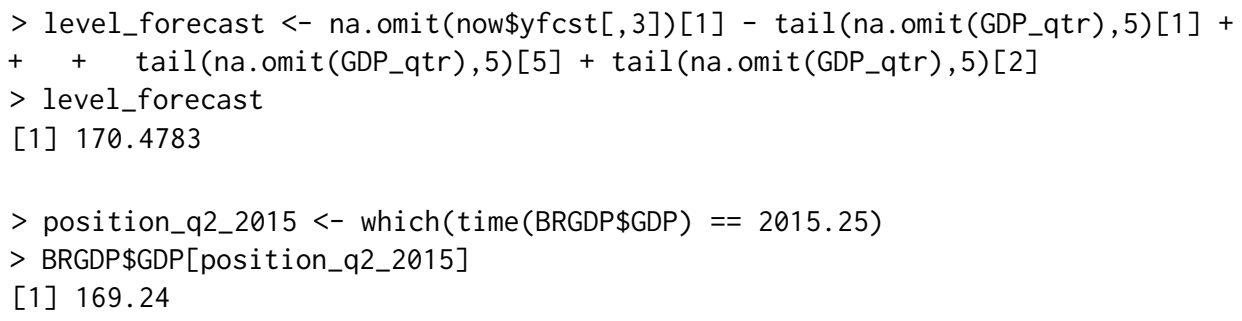

\section{A working example of the EM method:}

\section{The NY FED nowcast}

\section{Constructing the dataset}

In this example we work with the data the Federal Reserve of New York made available to reproduce its weekly nowcasting report ${ }^{4}$. The explanatory variables are mixed frequencies including both monthly and quarterly series.

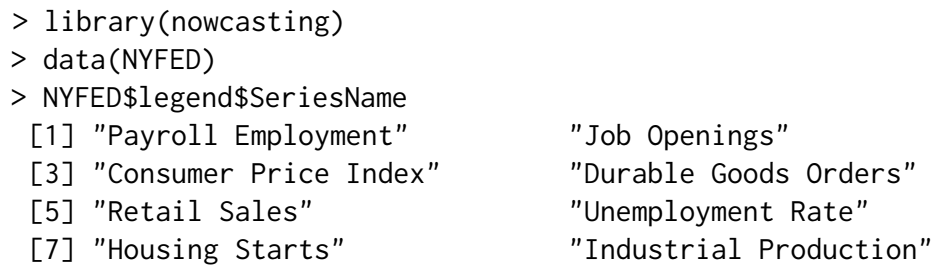

${ }^{4}$ https://www. newyorkfed.org/research/policy/nowcast 
[9] "Personal Income"

[11] "Imports"

[13] "Import Price Index"

[15] "Core PCE Price Index"

[17] "Building Permits"

[19] "Business Inventories"

[21] "Export Price Index"

[23] "Philadelphia Fed Mfg Index"

[25] "Real Gross Domestic Product"

\author{
"Exports" \\ "Construction Spending" \\ "Core Consumer Price Index" \\ "PCE Price Index" \\ "Capacity Utilization Rate" \\ "Unit Labor Cost" \\ "Empire State Mfg Index" \\ "Real Consumption Spending"
}

Similarly to the previous working example, the object NYFED contains all the necessary information to run the nowcast () function. The time series, the block structure, the transformations to make the variables stationary and the variables' frequencies can be loaded as illustrated below.

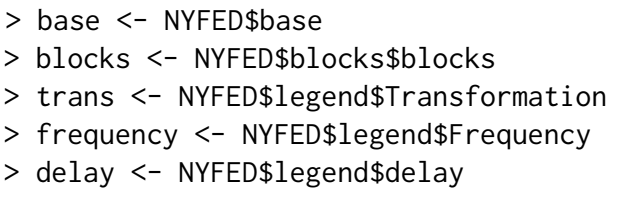

The dataset data can be prepared by using the function Bpanel(). Using the EM algorithm, there is no need to replace missing values that are not part of the jagged edges, as was the case with the Two-Stage method. This can be achieved by setting NA. replace to FALSE. In this case we do not want to discard series based on a particular ratio of missing values to total observations as was the case in the Two-Stage method. This is done by setting na.prop $=1$, where 1 indicates that only series with more than $100 \%$ missing values will be discarded.

$>$ data $<-$ Bpanel $($ base $=$ base, trans $=$ trans, NA.replace $=$ FALSE, na $\cdot$ prop $=1)$

\title{
Forecasts
}

The model's specifications are the same as those used by the NY FED. We therefore limit the number of factors, $r$, per block to one and define the factor process as a VAR(1), i.e., $p=1$. The convergence of the log-likelihood function is displayed every 5 iterations.

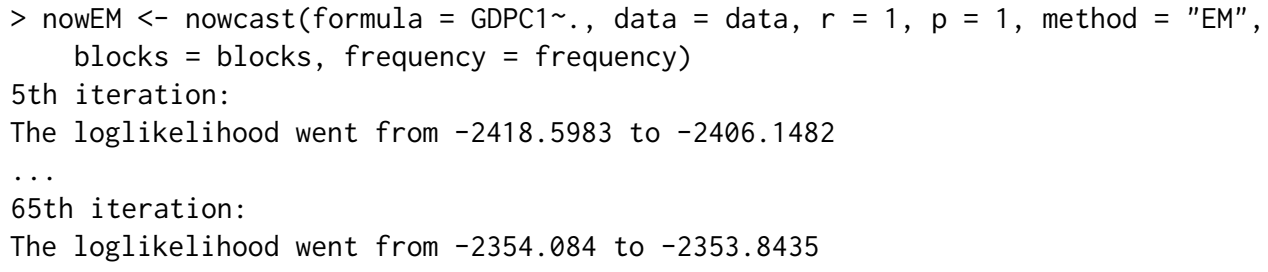

\section{Results}

Combining the functions nowcast() and PRTB() within a loop, we illustrate how a pseudo out-ofsample end-of-quarter nowcast can be made. The vector fcst_dates defines the last month of the quarters for which quarterly GDP growth will be nowcast. The vector delay contains approximate delays, in days, with which variables are published. This enables us to construct a pseudo real-time dataset for a given day.

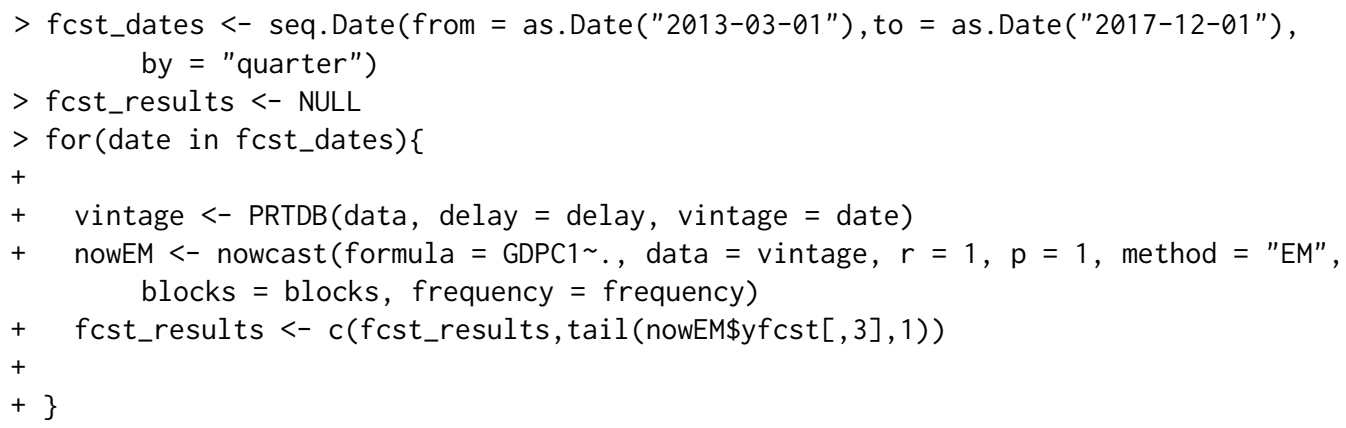


The results of this out-of-sample nowcast example, as well as the results of an out-of-sample ARIMA, are displayed below.

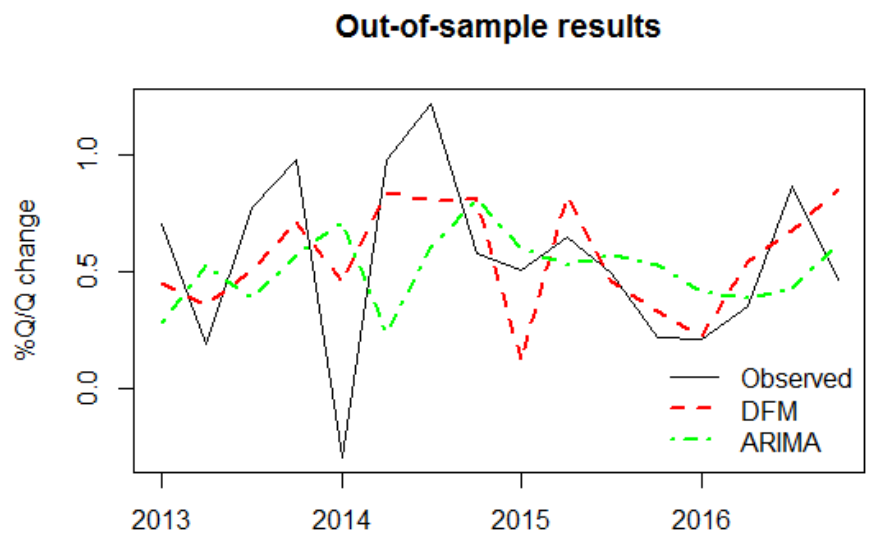

The root mean square prediction error can easily be calculated for the 2013-2016 period. For this given example, when compared to one-period-ahead projections given by an ARIMA model, a Theil's U statistic of 0.70 is obtained, signaling a $30 \%$ improvement over the benchmark.

\section{Summary}

The package nowcasting was developed in order to facilitate the use of dynamic factor models for large datasets as set out in Giannone et al. (2008) and Banbura et al. (2011). The package offers functions at each step of the forecasting process to help the user treat data, choose and estimate the value of parameters, as well as interpret results. We provided a working example for nowcasting Brazilian GDP, illustrating each step and showing how to implement the various functions available. We also used the New York FED nowcasting exercise to illustrate the EM algorithm. We will, in the future, work on adding new tools for the user to better leverage the EM method by identifying the source of forecast revisions. As shown by the New York FED nowcasting report, this is an interesting policy instrument that helps contextualizing forecast updates.

\section{Acknowledgements}

We thank Daniel Mesquita for revising some of the codes and our colleagues from FGV-IBRE for helpful inputs. We also thank an anonymous referee and the $\mathrm{R}$ journal editor Olivia Lau for constructive comments. The authors are responsible for any errors in this paper. This study was financed in part by the Coordenação de Aperfeiçoamento de Pessoal de Nível Superior - Brasil (CAPES) - Finance Code 001.

\section{Bibliography}

K. A. Aastveit and T. Trovik. Nowcasting norwegian gdp: The role of asset prices in a small open economy. Empirical Economics, 42(1):95-119, 2012. ISSN 1435-8921. URL https://doi . org/10.1007/ s00181-010-0429-9. [p1]

E. Angelini, G. Camba-Mendez, D. Giannone, L. Reichlin, and G. Rünstler. Short-Term Forecasts of Euro Area GDP Growth. Working Papers ECARES ECARES 2008-035, ULB - Universite Libre de Bruxelles, 2008. URL https://ideas.repec.org/p/eca/wpaper/2008_035.html. [p1]

J. Bai and S. Ng. Determining the number of factors in approximate factor models. Econometrica, 70(1): 191-221, 2002. URL https://doi.org/10.1111/1468-0262.00273. [p3, 6]

J. Bai and S. Ng. Determining the number of primitive shocks in factor models. Journal of Business $\mathcal{E}$ Economic Statistics, 25(1):52-60, 2007. URL https://doi .org/10.1198/073500106000000413. [p3, 7] 
M. Banbura, D. Giannone, and L. Reichlin. Nowcasting. Oxford Handbook on Economic Forecasting, 2011. [p1, 2, 3, 4, 5, 13]

M. Bańbura and M. Modugno. Maximum likelihood estimation of factor models on datasets with arbitrary pattern of missing data. Journal of Applied Econometrics, 29(1):133-160, 2014. URL https: //doi.org/10.1002/jae.2306. [p4]

M. Bańbura and G. Rünstler. A look into the factor model black box: Publication lags and the role of hard and soft data in forecasting gdp. International Journal of Forecasting, 27(2):333-346, 2011. URL https://doi.org/10.1016/j.ijforecast.2010.01.011. [p1]

J. Boivin and S. Ng. Are more data always better for factor analysis? Journal of Econometrics, 132(1): 169-194, 2006. URL https://doi.org/10.1016/j. jeconom.2005.01.027. [p1]

M. Chauvet. A monthly indicator of brazilian gdp. Brazilian Review of Econometrics, 21(1):1-47, 2001. URL https://doi.org/10.12660/bre.v21n12001.3191. [p1]

A. D'Agostino, G. Domenico, and P. Surico. (Un)Predictability and Macroeconomic Stability. Research Technical Papers 5/RT/06, Central Bank of Ireland, 2006. URL https://ideas. repec. org/p/cbi/ wpaper/5-rt-06.html. [p1]

A. D'Agostino, K. McQuinn, and D. O'Brien. Now-casting irish gdp. Research Technical Papers 9/RT/08, Central Bank of Ireland, 2008. URL https://doi .org/10.1787/19952899. [p1]

T. Dahlhaus, J.-D. Guenette, and G. Vasishtha. Nowcasting bric $+m$ in real time. Staff working papers, Bank of Canada, 2015. URL https://doi.org/10.1016/j.ijforecast. 2017.05.002. [p1]

J. Durbin and S. J. Koopman. Time Series Analysis by State Space Methods. Oxford University Press, 2 edition, 2012. URL https://EconPapers. repec.org/RePEc: oxp: obooks: 9780199641178. [p4]

M. Forni, M. Hallin, M. Lippi, and L. Reichlin. The generalized dynamic factor model consistency and rates. Journal of Econometrics, 119(2):231-255, 2004. URL https://doi .org/10.1016/s03044076(03)00196-9. [p1]

D. Giannone, L. Reichlin, and D. Small. Nowcasting: The real-time informational content of macroeconomic data. Journal of Monetary Economics, 55(4):665-676, 2008. URL https://doi org/10.1016/j. jmoneco.2008.05.010. [p1, 4, 5, 13]

M. Marcellino, J. Stock, and M. Watson. Macroeconomic forecasting in the euro area: Country specific versus area-wide information. European Economic Review, 47(1):1-18, 2003. URL https: //doi.org/10.1016/s0014-2921(02)00206-4. [p1]

R. S. Mariano and Y. Murasawa. A new coincident index of business cycles based on monthly and quarterly series. Journal of applied Econometrics, 18(4):427-443, 2003. URL https://doi .org/10. 1002/jae.695. [p2, 3, 4, 6]

T. D. Matheson. An Analysis of the Informational Content of New Zealand Data Releases: The Importance of Business Opinion Surveys. Economic Modelling, 27(1):304-314, 2010. URL https: //doi.org/10.1016/j. econmod.2009.09.010. [p1]

J. H. Stock and M. Watson. Dynamic factor models. Oxford Handbook on Economic Forecasting, 2011. [p4]

J. H. Stock and M. W. Watson. Forecasting with many predictors. Handbook of economic forecasting, 1 : 515-554, 2006. URL https://doi .org/10.1016/s1574-0706(05)01010-4. [p1]

J. H. Stock and M. W. Watson. Dynamic Factor Models, Factor-Augmented Vector Autoregressions, and Structural Vector Autoregressions in Macroeconomics, volume 2. Elsevier, 2016. URL https: //doi .org/ 10.1016/bs. hesmac. 2016.04.002. [p1,2]

F. Tusell. Kalman filtering in r. Journal of Statistical Software, Articles, 39(2):1-27, 2011. ISSN 1548-7660. URL https://doi.org/10.18637/jss.v039.i02. [p4]

C. Van Nieuwenhuyze, K. Ruth, A. Rua, P. Jelonek, A. Jakaitiene, A. Den Reijer, R. Cristadoro, G. Rünstler, S. Benk, and K. Barhoumi. Short-term forecasting of gdp using large monthly datasets: a pseudo real-time forecast evaluation exercise. Occasional Paper Series 84, European Central Bank, 2008. URL https://doi .org/10.1002/for.1105. [p1] 
Serge de Valk

EPGE Brazilian School of Economics and Finance (FGV EPGE)

60 Barão de Itambi, Botafogo, Rio de Janeiro - RJ

Brazil

serge.valk@fgv.br

Daiane Marcolino de Mattos

FGV-IBRE

60 Barão de Itambi, Botafogo, Rio de Janeiro - RJ

Brazil

daiane.mattos@fgv.br

Pedro Guilherme Costa Ferreira

FGV-IBRE

60 Barão de Itambi, Botafogo, Rio de Janeiro - RJ

Brazil

pedro.guilherme@fgv.br 